\title{
Effect of Exercise on $\mu$-Opioid Receptor Expression in the Rostral Ventromedial Medulla in Neuropathic Pain Rat Model
}

\author{
Young-Jin Kim, MD, Jeong-Hyun Byun, MD, In-Sung Choi, MD, PhD
}

\begin{abstract}
Department of Physical and Rehabilitation Medicine, Research Institute of Medical Sciences, Center for Aging and Geriatrics, and Regional Cardiocerebrovascular Center, Chonnam National University Hospital, Chonnam National University Medical School, Gwangju, Korea
\end{abstract}

Objective To investigate the effects of aerobic exercise on neuropathic pain and verify whether regular treadmill exercise alters opioid receptor expression in the rostral ventral medulla (RVM) in a neuropathic pain rat model.

Methods Thirty-two male Sprague-Dawley rats were used in the study. All rats were divided into 3 groups, i.e., group A, sham group ( $\mathrm{n}=10)$; group $\mathrm{B}$, chronic constriction injury (CCI) group $(\mathrm{n}=11)$; and group $\mathrm{C}$, CCI+exercise group $(n=11)$. Regular treadmill exercise was performed for 30 minutes a day, 5 days a week, for 4 weeks at the speed of $8 \mathrm{~m} / \mathrm{min}$ for 5 minutes, $11 \mathrm{~m} / \mathrm{min}$ for 5 minutes, and $22 \mathrm{~m} / \mathrm{min}$ for 20 minutes. Withdrawal threshold and withdrawal latency were measured before and after the regular exercise program. Immunohistochemistry and Western blots analyses were performed using antibodies against $\mu$-opioid receptor (MOR).

Results Body weight of group $\mathrm{C}$ was the lowest among all groups. Withdrawal thresholds and withdrawal latencies were increased with time in groups B and C. There were significant differences of withdrawal thresholds between group B and group C at 1st, 2nd, 3rd, and 4th weeks after exercise. There were significant differences of withdrawal latencies between group B and group C at 3rd and 4th weeks after exercise. MOR expression of group C was significantly decreased, as compared to that of group B in the RVM and spinal cord.

Conclusion In neuropathic pain, exercise induced analgesia could be mediated by desensitization of central MOR by endogenous opioids, leading to the shift of RVM circuitry balance to pain inhibition.

Keywords Exercise, Opioid receptors, Neuralgia, Medulla oblongata

Received August 27, 2014; Accepted October 10, 2014

Corresponding author: In-Sung Choi

Department of Physical and Rehabilitation Medicine, Chonnam National University Hospital, Chonnam National University Medical School, 42 Jebong-ro, Dong-gu, Gwangju 501-757, Korea

Tel: +82-62-220-5186, Fax: +82-62-228-5975, E-mail: drchoiis@hanmail. net

() This is an open-access article distributed under the terms of the Creative Commons Attribution Non-Commercial License (http://creativecommons. org/licenses/by-nc/4.0) which permits unrestricted noncommercial use, distribution, and reproduction in any medium, provided the original work is properly cited.

Copyright $\odot 2015$ by Korean Academy of Rehabilitation Medicine

\section{INTRODUCTION}

Neuropathic pain has been defined as pain caused by a lesion or disease of the somatosensory nervous system [1]. Neuropathic pain can lead to numerous symptoms, such as spontaneous pain, allodynia, and hyperalgesia. Activation of peripheral nociceptive nerve fibers modifies the nerve's response to further stimuli. Nociceptive stimulation results in the release of neurotransmitters and peptides that increase the excitability or sensitize 
sensory neurons (peripheral sensitization) [2]. Increased input from peripheral nociceptive neurons leads to alterations in the spinal cord dorsal horn, including disinhibition and increased responsiveness of central neurons (central sensitization) [3]. Both peripheral and central processes contribute to the induction of neuropathic pain. A change in the function, chemistry, and structure of neurons clearly underlies the production of stimulusevoked pain. These changes are observed in the sensory neurons, spinal cord, and cortex of brain [2].

Neuropathic pain is chronic in most patients [4]. In addition, neuropathic pain is often difficult to treat. Existing pharmacologic treatments for neuropathic pain are limited, with no more than $40 \%-60 \%$ of patients obtaining partial relief of pain [5]. Therefore, there is growing attention to non-pharmacologic managements for neuropathic pain.

Regular exercise has beneficial effects on physical and mental health, such as depression and stress reduction, mood elevation and reduced pain perception [6-8]. Exercise may serve as an alternative for effective and healthy pain management [9]. Activation of the endogenous opioid system may be responsible for the analgesic response following exercise [10]. Endogenous opioids may act by modulating pain at peripheral and central levels [11]. Few studies have examined central opioid activity after exercise $[12,13]$.

Rostral ventromedial medulla (RVM) is critical for the modulation of nociceptive transmission [14,15]. RVM facilitates rather than suppresses spinal nociceptive processing via descending projections after peripheral nerve injury [16]. However, alterations of brain opioid receptors after exercise are poorly studied $[17,18]$.

The aim of this study was to investigate the effects of aerobic exercise on neuropathic pain and verify whether regular treadmill exercise alters the $\mu$-opioid receptor (MOR) expression in RVM in a neuropathic pain rat model.

\section{MATERIALS AND METHODS}

\section{Materials}

Thirty-two male Sprague-Dawley rats, weighing 250-300 $\mathrm{g}$, aged 10 weeks old, were used. The rats were housed at a temperature of $22^{\circ} \mathrm{C} \pm 2^{\circ} \mathrm{C}$ and a humidity of $55 \%$ with a 12-hour light-dark cycle (light between 8 AM to $8 \mathrm{PM}$ ) and had free access to food and water ad libitum.
Ten rats received sham operation. Twenty-two rats underwent chronic constriction injury (CCI) procedure [19]. CCI rats were randomly divided into 2 groups according to the application of regular treadmill exercise program. All 3 groups were as follows: group $A(n=10)$, sham group; group B ( $\mathrm{n}=11)$, CCI group; and group C $(n=11)$, CCI+regular treadmill exercise group. All animal experiments were conducted in accordance with the guidelines of the Chonnam National University Hospital Animal Care and Use Committee.

\section{Induction of sciatic neuropathy}

Unilateral CCI of right sciatic nerve was induced as described by Bennett and Xie [19]. All rats were anesthetized with intraperitoneal injection of $50 \mathrm{mg} / \mathrm{kg}$ of tiletamine $\mathrm{HCl}$ /zolazepam $\mathrm{HCl}$, Zoletil (Virbac, Carros, France) and $7 \mathrm{mg} / \mathrm{kg}$ of xylazine, Rompun (Bayer Korea, Seoul, Korea). We clipped the hair in the right gluteal region and disinfected the skin with povidone iodine. The skin was opened over a length of $2 \mathrm{~cm}$ in the proximal half of the line between trochanter and knee joint. The biceps femoris muscle was longitudinally separated and the sciatic nerve was exposed under the muscle. Proximal to the trifurcation of sciatic nerve, 4 loose ligatures of 4-0 chromic catgut at an interval of $1 \mathrm{~mm}$ were placed around the sciatic nerve. After the induction of CCI, the skin and muscle fascia were sutured. For the prevention of infection, $5 \mathrm{mg} / \mathrm{kg}$ of gentamicin sulfate was administered into the gluteal muscle for 3 days. Sham operation followed the same procedures except loose ligation of sciatic nerve. All surgical procedures were performed by a researcher.

\section{Regular treadmill exercise program}

Only group $\mathrm{C}$ underwent regular treadmill exercise program. Treadmill exercise was conducted using an electric treadmill machine, Exer-3/6 (Columbus Instruments, Columbus, OH, USA) for 40 minutes a day, 5 days per week, for 4 weeks. Regular treadmill exercise program was composed of warm-up for 5 minutes, main exercise for 30 minutes, and cool-down for 5 minutes. Main exercise was $8 \mathrm{~m} / \mathrm{min}$ for 5 minutes, $11 \mathrm{~m} / \mathrm{min}$ for 5 minutes, and $22 \mathrm{~m} / \mathrm{min}$ for 20 minutes [20].

\section{Evaluation of thermal hyperalgesia and mechanical allodynia \\ Withdrawal frequency and withdrawal latency were}


evaluated on the 10th day after CCI surgery in all rats. Withdrawal frequency and withdrawal latency were measured serially after regular treadmill exercise program (every week, for 4 weeks). For the evaluation of mechanical allodynia, withdrawal frequency of the hind paw was measured by determining paw withdrawal after probing the paw with a series of calibrated monofilaments, Touch Test Sensory Evaluator (North Coast Medical Inc., Gilroy, CA, USA) [21]. All rats were placed on a metal mesh and the plantar surface of the operated hind paw was touched with monofilaments. The withdrawal threshold was determined by sequentially increasing and decreasing the stimulus strength, and analyzed using a Dixon nonparametric test. Tests were conducted twice in each rat. Data were expressed as the mean withdrawal threshold.

For the evaluation of thermal hyperalgesia, withdrawal latency to the heat stimulus was measured by paw withdrawal from a focused beam of radiant heat, Plantar Test (Ugo Basile, Varese, Italy) onto the surface of the hind paw [22]. Prior to testing, all rats were placed in a plastic box with a 3-mm-thick glass floor to acclimate to their environment. A mobile radiant infrared source under the transparent glass floor was applied to the plantar surface of right hind paw. Withdrawal latency was defined as the time from the onset of the heat stimulus to the withdrawal of the hind paw. The intensity of the heat stimulus was 55. A cutoff time of 30 seconds was set to avoid possible tissue damage. Each paw was tested twice with at least 5 minutes interval between consecutive tests. The mean value of the 2 trials was used for data analysis.

\section{Immunohistochemistry}

The sampled rats of each group were sacrificed after regular treadmill exercise program. The heart was exposed under deep anesthesia with $2.0 \%$ Forane. Each rat was perfused transcardially with $100 \mathrm{~mL}$ saline, followed by $400 \mathrm{~mL} 4 \%$ paraformaldehyde phosphate buffer (0.1 $\mathrm{mol} / \mathrm{L} \mathrm{PB}, \mathrm{pH}$ 7.4). The spinal cords ( $2 \mathrm{~cm}$ long) were removed from the lumbar enlargement, fixed in $10 \%$ formalin solution for 24 hours, and embedded in paraffin wax. RVM was isolated at $11 \mathrm{~mm}$ posterior to the bregma [23]. Paraffin embedding tissues were cut axially at $4 \mu \mathrm{m}$ thickness. After being posted on the slide, tissues were treated with xylene and deparaffinized. The sections were dehydrated in graded alcohol. After rinsing with TBS$\mathrm{T}$ saline (Tris-Buffered Saline, $\mathrm{pH}$ 7.4, Tween20), nerve sections were incubated with $3 \%$ hydrogen peroxide for 30 minutes. Subsequently, they were incubated first with $1 \%$ pre-immune BSA to decrease non-specific staining and then reacted with the primary antibody. Sections were incubated with 1:200 primary antibody solution, i.e., anti-MOR antibody (abcam, Cambridge, MA, USA) as. Incubations with the primary antibody were conducted overnight at $4^{\circ} \mathrm{C}$. Subsequently, sections were washed 5 times with TBS-T followed by incubation with quick antirabbit immunoglobulin for 60 minutes. Sections were then washed 5 times with TBS-T, followed by incubation with streptavidin peroxidase for 10 minutes. The same sections were washed and visualized with 3,3'-diaminobenzidine (DAB) for 10 minutes and counterstained with Mayer's hematoxylin. The expression of MOR was examined under a light microscope (Nikon, Tokyo, Japan) at a magnification of 200 .

\section{Western blot analysis}

Rats were rapidly decapitated on intraperitoneal anesthesia. RVM and spinal cord were removed and immediately placed on liquid nitrogen and stored at $-70^{\circ} \mathrm{C}$ until use. Rapidly frozen sections were then washed with phosphate-buffered saline (PBS). Washed tissues were manually homogenized with liquid nitrogen to a powder form, and placed in a 1.5-mL tube. Extraction buffer, PRO-PREP Protein Purification kit (PRO-PREP Protein Extraction Solution) $500 \mu \mathrm{L}$ solution was added and dispersed. Samples were agitated, followed by 30-minute centrifugation at $12,000 \mathrm{rpm}$ at $4^{\circ} \mathrm{C}$ to separate and $200 \mu \mathrm{L}$ supernatant was transferred to a new tube. Protein was diluted and quantified on ELISA plates with the Pierce BCA Protein Assay Kit (Thermo Fisher Scientific Inc., Waltham, MA, USA). Protein denaturation was induced by heating for 5 minutes at $38^{\circ} \mathrm{C}$. Denatured proteins were separated by electrophoresis on $12 \%$ acrylamide gel and electrotransferred to a polyvinylidene difluoride membrane at $300 \mathrm{~mA}$ for 1 hour. The membranes were blocked in 3\% skim milk/TBS-T for 1 hour at room temperature and MOR antibody was incubated overnight at 4oC. Membranes were washed in Tris-buffered saline with $0.1 \%$ Tween-20 (TBS-T) and incubated for 1 hour at room temperature with goat anti-rabbit IgG-HRP (1:2500 in TBS-T; Santa Cruz Biotechnology Inc., Santa Cruz, CA, USA). After washing TBS-T, immunoreactive protein bands were visualized using the enhanced chemilumi- 
nescence kit (Immobilon Western Chemiluminescent Kit; Merck Millipore, Billerica, MA, USA) and LAS-3000 Imaging System (Fujifilm, Tokyo, Japan). After the images of bands were scanned in grayscale at a resolution of 600 dpi, the bands were quantified using ImageJ (National Institute of Health, Bethesda, MD, USA).

\section{Statistical analysis}

Statistical analyses were performed using SPSS statistical software ver. 21 (IBM SPSS, Armonk, NY, USA). The results were expressed as mean \pm standard deviation. Repeated measures analysis of variance (RM-ANOVA) was used to study within-subjects effects with time. Post-hoc testing consisted of multiple comparisons using the Bonferroni method. Independent samples t-test was used to compare the results of behavioral tests between the 2 groups. Two-way repeated measures analysis of variance (ANOVA) was used to analyze the differences among groups and the changes with time. Post-hoc testing consisted of a pairwise comparison using the Bonferroni method for multiple comparisons. ANOVA was used to compare the expression of MOR protein among groups. Bonferroni method was chosen for Post-hoc analysis. Spearman correlation analysis was used to study correlation between MOR expression and behavioral tests. A difference was accepted as significant if was $p<0.05$.

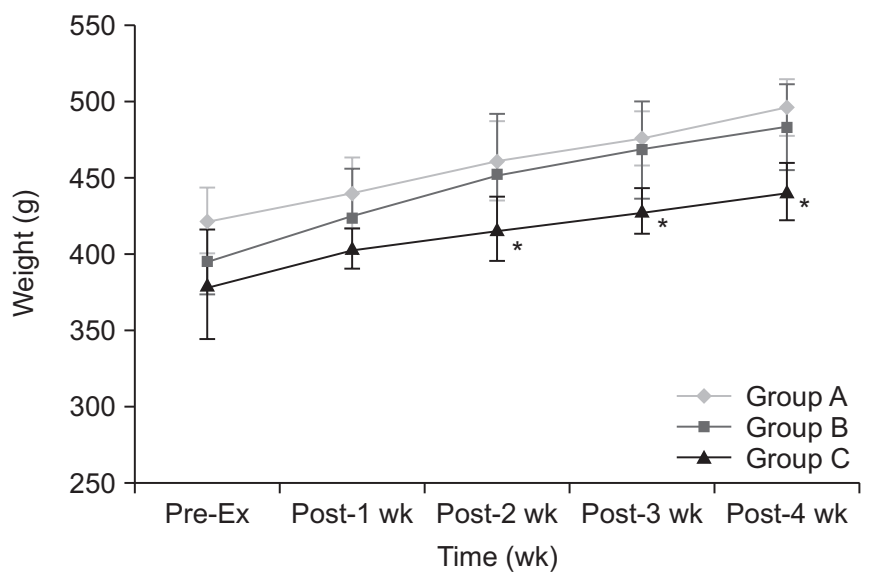

Fig. 1. The changes of body weight with time. There is increase in body weight with time in all groups. Body weight of group $\mathrm{C}$ was the lowest in all groups. There are significant differences of body weight between no exercise-CCI group and exercised CCI group at 2nd, 3rd, and 4th weeks after the beginning of the regular exercise program. ${ }^{*}$ p $<0.05$ compared to group B. CCI, chronic constriction injury.

\section{RESULTS}

There were no significant differences between group B and group $C$ in pre-exercise weight ( $\mathrm{p}=0.266)$, withdrawal threshold ( $\mathrm{p}=0.733)$, and withdrawal latency $(\mathrm{p}=0.894)$, respectively.

\section{Body weight}

Body weight increased with time in all groups (Fig. 1). Body weight of group $\mathrm{C}$ was the lowest among all groups. There was within-subjects effect $(\mathrm{p}=0.000)$. Within-subjects difference was significant after 2 weeks of treadmill exercise $(p=0.001)$. In addition, there was between-subjects effect $(\mathrm{p}=0.000)$. In pairwise comparisons, the mean difference was not significant between group A and B ( $p=0.148)$, but the mean differences were significant between group $B$ and group $C(p=0.039)$ and between group $A$ and group $C(p=0.001)$. Treadmill exercise changed the body weight in group $\mathrm{C}$, as compared to group $\mathrm{A}(\mathrm{p}=0.000)$ and group $B(p=0.004)$. There were significant differences of body weight between group $B$ and group $C$ at 2nd $(\mathrm{p}=0.017), 3 \mathrm{rd}(\mathrm{p}=0.003), 4$ th $(\mathrm{p}=0.001)$ weeks after the beginning of the regular exercise program. There was no significant interaction between time and group for weight $(\mathrm{p}=0.214)$.

\section{Mechanical allodynia}

Withdrawal thresholds to mechanical stimuli in-

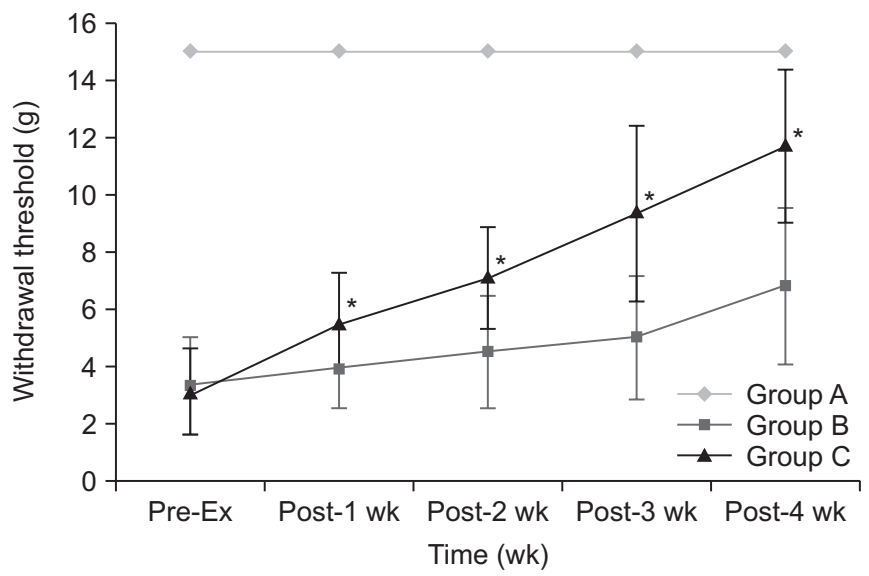

Fig. 2. The changes of withdrawal threshold to mechanical stimuli with time. Withdrawal thresholds are increased in group C, as compared to group B at every week after the beginning of the regular exercise program. ${ }^{*} \mathrm{p}<0.05$ compared to group B. 
creased with time in groups B and C (Fig. 2). There was within-subjects effect $(p=0.000)$. Within-subjects difference was significant after 2 weeks of treadmill exercise $(p=0.001)$. Furthermore, there was between-subjects effect $(\mathrm{p}=0.000)$. In pairwise comparisons, the mean differences were significant between groups $A$ and $B(p=0.000)$, between groups $B$ and $C(p=0.018)$ and between groups $A$ and $C(p=0.000)$. Treadmill exercise increased withdrawal thresholds in group $\mathrm{C}$, as compared to group $\mathrm{A}(\mathrm{p}=0.000)$ and group $B(p=0.000)$. There were significant differences of withdrawal threshold between groups $\mathrm{A}$ and $\mathrm{C}$ at every time point. There were significant increase of withdrawal thresholds between group B and group $C$ at 1 st $(p=0.037)$, 2nd ( $\mathrm{p}=0.006)$, 3rd $(\mathrm{p}=0.002)$, and 4 th $(\mathrm{p}=0.001)$ weeks after the onset of the regular exercise program. There was significant interaction between time and group for withdrawal threshold $(\mathrm{p}=0.000)$. Withdrawal threshold was inversely correlated with MOR expression in RVM $(\mathrm{p}=0.003, \mathrm{r}=-0.520)$ and spinal cord $(\mathrm{p}=0.000, \mathrm{r}=-0.647)$.

\section{Thermal hyperalgesia}

Withdrawal latencies to noxious thermal stimuli increased with time in groups B and C (Fig. 3). There was within-subjects effect $(p=0.000)$. Within-subjects difference was significant after 3 weeks of treadmill exercise $(p=0.009)$. There was between-subjects effect $(p=0.000)$. In pairwise comparisons, the mean differences were significant between groups A and B $(p=0.000)$, between groups $B$ and $C(p=0.003)$, and between groups $A$ and $C(p=0.000)$. Treadmill exercise increased withdrawal latencies in group $\mathrm{C}$, as compared to group $\mathrm{A}(\mathrm{p}=0.000)$ and group $B(p=0.029)$. There were significant differences of withdrawal threshold between group $\mathrm{A}$ and group $\mathrm{C}$ at every time point. There were significant increase of withdrawal latencies between group $\mathrm{B}$ and group $\mathrm{C}$ at 3rd ( $\mathrm{p}=0.007)$ and 4 th $(\mathrm{p}=0.010)$ weeks after the beginning of the regular exercise program. There was no significant interaction between time and group for withdrawal thresh-

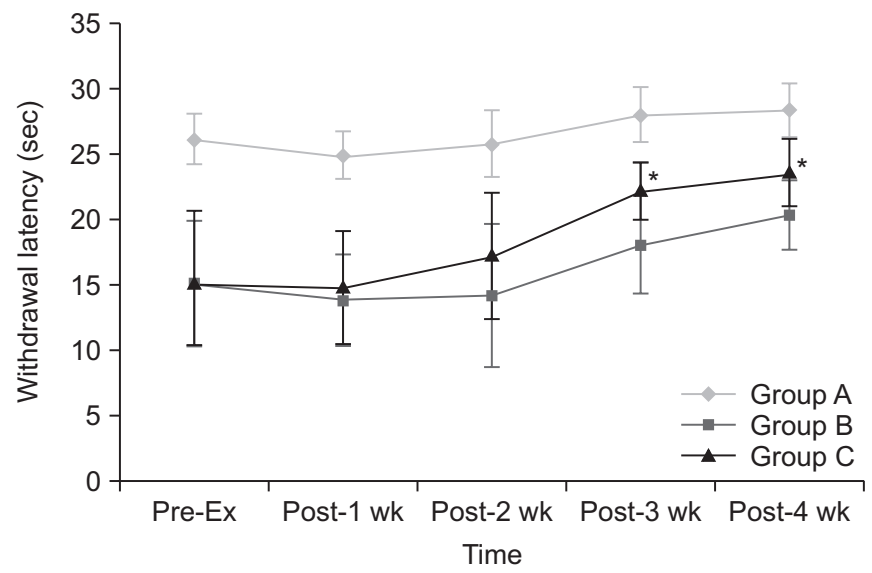

Fig. 3. The changes of withdrawal latency to noxious heat stimuli with time. Withdrawal latencies are increased in group C, as compared to group B at 3rd and 4th weeks after the beginning of the regular exercise program. ${ }^{*} \mathrm{p}<0.05$ compared to group B.
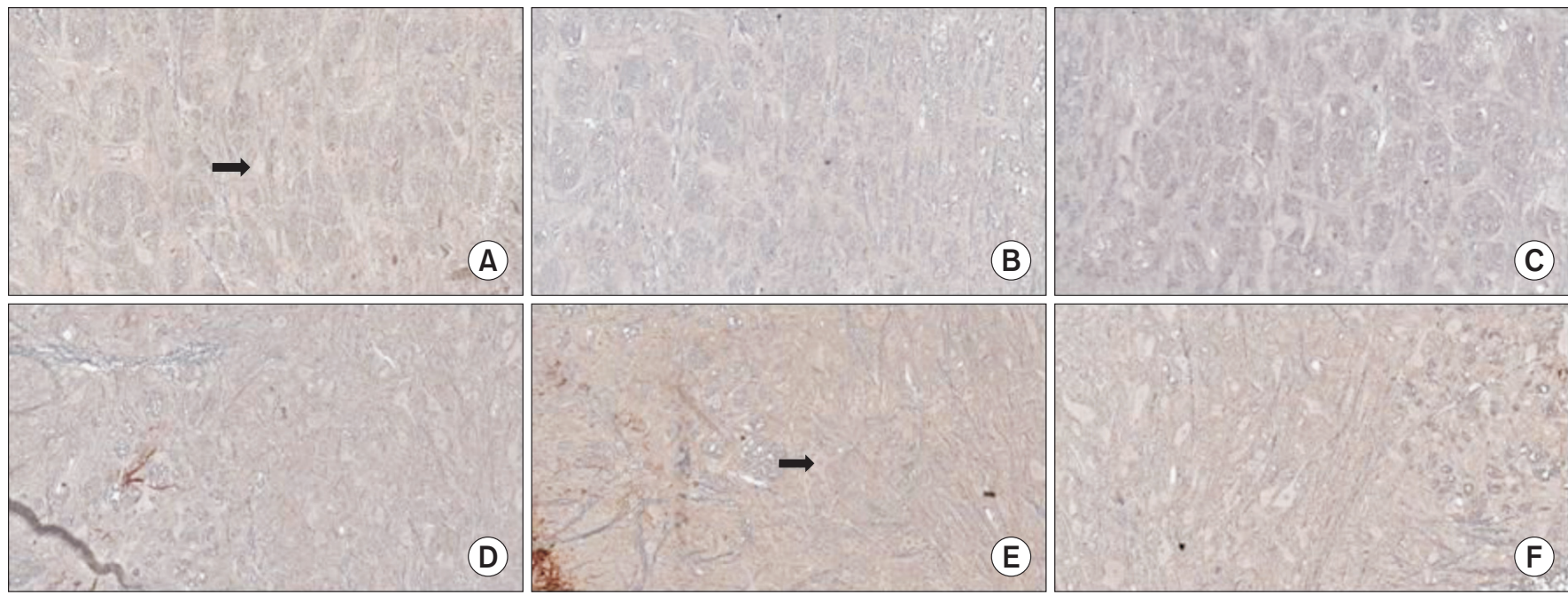

Fig. 4. Immunohistochemistry findings in the rostral ventromedial medulla (RVM, upper panel) and spinal cord (lower panel): (A, D) group A, (B, E) group B, (C, F) group C. Abundant stained neuronal perikarya (black arrow) are seen in the RVM and spinal cord $(\times 200)$. 
(A)

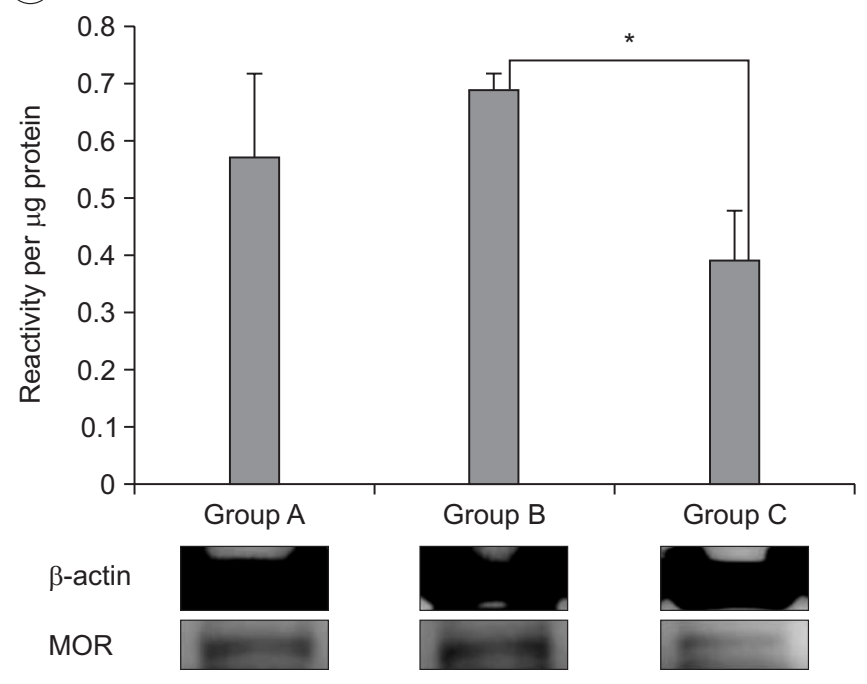

(B)

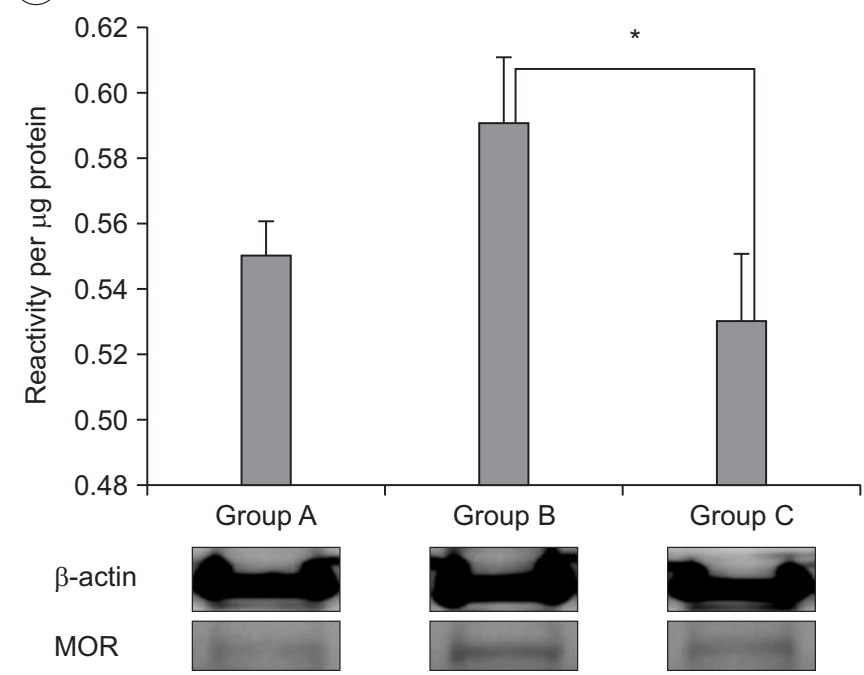

Fig. 5. The expression of $\mu$-opioid receptor (MOR) by Western blots analysis. MOR protein expression of group C is significantly lower than that of group B in rostral ventromedial medulla (A) and spinal cord (B).

old $(\mathrm{p}=0.054)$. Withdrawal latency was inversely correlated with MOR expression in RVM ( $\mathrm{p}=0.043, \mathrm{r}=-0.365)$ and spinal cord $(\mathrm{p}=0.014, \mathrm{r}=-0.439)$.

\section{MOR reactivity}

MOR immunoreactive neuronal perikarya were seen in the RVM and spinal cord (Fig. 4). MOR expression was significantly decreased in group $\mathrm{C}$, as compared to group $B$ in the RVM ( $p=0.033)$ and spinal cord $(p=0.030)$, respectively (Fig. 5). Post-hoc tests did not show any significant difference in RVM between group $A$ and group $B$ $(\mathrm{p}=0.548)$ and between group $A$ and group $C(p=0.233)$. Post-hoc tests did not show any significant difference between group $A$ and group $B(p=0.094)$ and between group $A$ and group $C(p=1.000)$ in the spinal cord.

\section{DISCUSSION}

Neuropathic pain can result from peripheral nerve injuries, which trigger maladaptive alterations in the nervous system leading to peripheral and central sensitization that underlie transition to chronic pain [24]. Regular exercise improves neuropathic pain. Rhythmic, weightbearing exercise reverses signs of neuropathic pain in the spinal cord injury rat model [25]. Extended swimming reduces inflammatory and peripheral neuropathic pain [26]. Short-lasting treadmill training for 5 days reportedly reduces CCI-induced neuropathic pain in mice and facil- itates the regenerative processes of the injured nerve [27]. Regular moderate-intensity treadmill exercise decreases sensory hypersensitivity in neuropathic pain rats [12]. Similar to the previous reports $[12,26,27]$, exercised CCI rats showed improved neuropathic pain symptoms in this study. Besides, body weights of CCI rats with regular treadmill exercise program for 4 weeks were lower than those of non-exercised rats.

Swimming has been the predominant exercise stimulus in most of the previous experimental studies [10]. The exercise stressor is important in determining which of the opioid and non-opioid analgesia systems are activated during exercise. Because of the question of whether analgesia following swimming is a function of the forced nature of the swim and to avoid the effect of water temperature on analgesia in swimming [10], we used treadmill running as the exercise stimulus in this study. Furthermore, it is difficult to make experimental animals do high-intensity treadmill exercise or extended swimming exercise. Long-lasting, regular, moderate-intensity exercise programs are preferable in terms of therapeutic effects of exercise.

Exercise-induced analgesia is mediated by the endogenous opioid system. Previous studies have shown an increased plasma level of $\beta$-endorphin during and after aerobic exercise [28]. Regular running exercise increases cerebrospinal fluid content of endogenous opioids [29]. Exercise effects in neuropathic pain may be mediated by 
an increase in the endogenous opioids content or release in the brain [12]. Regular moderate-intensity treadmill exercise decreases tactile and thermal hypersensitivity in neuropathic pain by increasing the expression of endogenous brainstem opioids. The contents of $\beta$-endorphin and met-enkephalin in the periaqueductal gray area (PAG) and RVM are increased in exercise-trained spinal nerve ligation (SNL)-induced neuropathic pain rats. Naloxone reverses the effects of exercise, suggesting that the activity of endogenous opioids is necessary for exercise training-mediated reversal of SNL-induced sensory hypersensitivity [12]. Analgesic effect of exercise is commonly explained by the $\beta$-endorphin hypothesis [30]. However, indirect measurement of increased $\beta$-endorphin in peripheral blood and cerebrospinal fluid does not reflect the opioid level in the central nervous system (CNS) $[18,31]$. Plasma $\beta$-endorphin level is not correlated with the subject's pain threshold [32]. Sforzo et al. [18] also suggested that exercise-induced enhancement of peripheral $\beta$-endorphin does not have a supraspinal action and prolonged swimming alters opioid receptor occupancy in the rat brain. We investigated MOR expression in the CNS instead of the $\beta$-endorphin level.

Opioid receptor is targeted for the treatment of pain related disorders. The endogenous opioid peptides bind to 3 primary opioid receptor types (MOR, $\delta$-opioid receptor, $\kappa$-opioid receptor) that mediate analgesia. The most commonly used opioids for pain management act on MOR systems [33]. Classically, MORs are presynaptic and inhibit neurotransmitter release; through this mechanism, they inhibit the release of the inhibitory neurotransmitter GABA and decrease the inhibition of dopamine pathways, causing more dopamine to be released [34].

Opioid receptors are expressed in pain-modulating descending pathways that include the medulla, locus ceruleus and PAG. The activation of opioid receptors at these locations directly inhibits neurons, which in turn inhibit spinal cord pain transmission [35]. RVM is an important component of the descending nociceptive system that constitutes a major mechanism in the control of pain transmission [36-38]. RVM has both descending pain inhibitory, as well as descending pain facilitatory functions [15]. Within the RVM region, 3 different populations of neurons including on-cell, off-cell, and neutral cells are identified [39]. Decreased firing of off-cells inhibits nociceptive processing. Increased activity of on-cells promotes nociception. The shift in the balance of RVM circuitry during neuropathic pain is such that on-cell activity predominates over that of off-cells and overall RVM has pain facilitatory influence [39]. Pain facilitatory on-cells of the RVM reportedly express MOR [40]. A subset of RVM neurons expressing MORs contribute to the maintenance of thermal hyperalgesia in neuropathic pain [16]. The selective lesioning of MOR expressing RVM neurons by dermorphin-saporin conjugate reverses abnormal pain states in the injured hindpaws in unilateral SNL models [15]. De Oliveira et al. [17] reported that acute and chronic exercise modulates MOR expression in the hippocampal formation of rats. A previous study showed thata few sessions of exercise (acute exercise) increase MOR expression in the hippocampal formation and this effect is reversed by long-term exercise (chronic exercise) [17]. Chronic exercise leads to the development of MOR opioid tolerance and physical dependence (compensatory down-regulation of opioid receptors during exercise) [41]. There is a lack of information concerning the alteration of central opioid receptor after exercise in neuropathic pain [42]. Our study showed that MOR-like immunoreactive RVM neurons were slightly increased in CCI rats, as compared with intact rats. Kainic acid microinjection into the RVM in the CCI rats significantly reduces MOR-like immunoreactive neurons in the RVM and increases paw withdrawal latency indicative of attenuation of thermal hyperalgesia [16]. Furthermore, MOR mRNA expression in the spinal cord of sciatic nerve-ligated mice is increased, as compared with sham-operated mice [43]. In this study, neuropathic pain improved and MOR expressions of RVM and spinal cord were decreased after regular treadmill exercise program.

Our data suggested that analgesic effect of exercise in neuropathic pain is mediated by desensitization of central MOR and the shift of RVM circuitry balance to decreased on-cell activity. Further verification requires neuronal recording using microelectrodes.

Opioid analgesics are successful in management of neuropathic pain. Combination of opioid analgesics and exercise could decrease the dose of opioid analgesics because of similar action mechanisms. Further investigation of opioid receptor activity associated with exercise is needed. Neuroimaging studies could be helpful in expanding our understanding of the brain circuitry in- 
volved in exercise-induced analgesia.

\section{CONFLICT OF INTEREST}

No potential conflict of interest relevant to this article was reported.

\section{ACKNOWLEDGMENTS}

This study was supported by a grant (No. CRI 11 001) Chonnam National University Hospital Biomedical Research Institute.

\section{REFERENCES}

1. Merskey H, Bogduk N. Classification of chronic pain: descriptions of chronic pain syndromes and definitions of pain terms. 2nd ed. Seattle: International Association for the Study of Pain; 2012.

2. Woolf CJ, Mannion RJ. Neuropathic pain: aetiology, symptoms, mechanisms, and management. Lancet 1999;353:1959-64.

3. Bennett GJ, Kajander KC, Sahara Y, Iadarola MJ, Sugimoto T. Neurochemical and anatomical changes in the dorsal horn of rats with an experimental painful peripheral neuropathy. In: Cervero F, Bennett GJ, Headley PM, editors. Processing of sensory information in the superficial dorsal horn of the spinal cord. New York: Plenum Press; 1989. p. 463-71.

4. Dworkin RH. An overview of neuropathic pain: syndromes, symptoms, signs, and several mechanisms. Clin J Pain 2002;18:343-9.

5. Dworkin RH, O'Connor AB, Backonja M, Farrar JT, Finnerup NB, Jensen TS, et al. Pharmacologic management of neuropathic pain: evidence-based recommendations. Pain 2007;132:237-51.

6. Rosch PJ. Exercise and stress reduction. Compr Ther 1985;11:10-5.

7. Wildmann J, Kruger A, Schmole M, Niemann J, Matthaei $\mathrm{H}$. Increase of circulating beta-endorphin-like immunoreactivity correlates with the change in feeling of pleasantness after running. Life Sci 1986;38:9971003.

8. Janal MN, Colt EW, Clark WC, Glusman M. Pain sensitivity, mood and plasma endocrine levels in man following long-distance running: effects of naloxone.
Pain 1984;19:13-25.

9. Galdino GS, Duarte ID, Perez AC. Participation of endogenous opioids in the antinociception induced by resistance exercise in rats. Braz J Med Biol Res 2010;43:906-9.

10. Koltyn KF. Analgesia following exercise: a review. Sports Med 2000;29:85-98.

11. Schmitt TK, Mousa SA, Brack A, Schmidt DK, Rittner HL, Welte $M$, et al. Modulation of peripheral endogenous opioid analgesia by central afferent blockade. Anesthesiology 2003;98:195-202.

12. Stagg NJ, Mata HP, Ibrahim MM, Henriksen EJ, Porreca F, Vanderah TW, et al. Regular exercise reverses sensory hypersensitivity in a rat neuropathic pain model: role of endogenous opioids. Anesthesiology 2011;114:940-8.

13. Werme M, Thoren P, Olson L, Brene S. Running and cocaine both upregulate dynorphin mRNA in medial caudate putamen. Eur J Neurosci 2000;12:2967-74.

14. Leong ML, Gu M, Speltz-Paiz R, Stahura EI, Mottey $\mathrm{N}$, Steer CJ, et al. Neuronal loss in the rostral ventromedial medulla in a rat model of neuropathic pain. J Neurosci 2011;31:17028-39.

15. Porreca F, Burgess SE, Gardell LR, Vanderah TW, Malan TP Jr, Ossipov MH, et al. Inhibition of neuropathic pain by selective ablation of brainstem medullary cells expressing the mu-opioid receptor. J Neurosci 2001;21:5281-8.

16. Mase H, Sakai A, Sakamoto A, Suzuki H. A subset of $\mu$-opioid receptor-expressing cells in the rostral ventromedial medulla contribute to thermal hyperalgesia in experimental neuropathic pain. Neurosci Res 2011;70:35-43.

17. De Oliveira MS, da Silva Fernandes MJ, Scorza FA, Persike DS, Scorza CA, da Ponte JB, et al. Acute and chronic exercise modulates the expression of MOR opioid receptors in the hippocampal formation of rats. Brain Res Bull 2010;83:278-83.

18. Sforzo GA, Seeger TF, Pert CB, Pert A, Dotson CO. In vivo opioid receptor occupation in the rat brain following exercise. Med Sci Sports Exerc 1986;18:380-4.

19. Bennett GJ, Xie YK. A peripheral mononeuropathy in rat that produces disorders of pain sensation like those seen in man. Pain 1988;33:87-107.

20. Kim YP, Kim HB, Jang MH, Lim BV, Kim YJ, Kim H, et al. Magnitude- and time-dependence of the effect of 
treadmill exercise on cell proliferation in the dentate gyrus of rats. Int J Sports Med 2003;24:114-7.

21. Chaplan SR, Bach FW, Pogrel JW, Chung JM, Yaksh TL. Quantitative assessment of tactile allodynia in the rat paw. J Neurosci Methods 1994;53:55-63.

22. Hargreaves K, Dubner R, Brown F, Flores C, Joris J. A new and sensitive method for measuring thermal nociception in cutaneous hyperalgesia. Pain 1988;32:7788.

23. Paxinos G, Watson C. The rat brain in stereotaxic coordinates. 7th ed. Burlington: Elsevier Science; 2013.

24. Costigan M, Scholz J, Woolf CJ. Neuropathic pain: a maladaptive response of the nervous system to damage. Annu Rev Neurosci 2009;32:1-32.

25. Hutchinson KJ, Gomez-Pinilla F, Crowe MJ, Ying Z, Basso DM. Three exercise paradigms differentially improve sensory recovery after spinal cord contusion in rats. Brain 2004;127(Pt 6):1403-14.

26. Kuphal KE, Fibuch EE, Taylor BK. Extended swimming exercise reduces inflammatory and peripheral neuropathic pain in rodents. J Pain 2007;8:989-97.

27. Cobianchi S, Marinelli S, Florenzano F, Pavone F, Luvisetto S. Short- but not long-lasting treadmill running reduces allodynia and improves functional recovery after peripheral nerve injury. Neuroscience 2010;168:273-87.

28. Bement MK, Sluka KA. Low-intensity exercise reverses chronic muscle pain in the rat in a naloxone-dependent manner. Arch Phys Med Rehabil 2005;86:173640.

29. Hoffmann P, Terenius L, Thoren P. Cerebrospinal fluid immunoreactive beta-endorphin concentration is increased by voluntary exercise in the spontaneously hypertensive rat. Regul Pept 1990;28:233-9.

30. Morgan WP. Affective beneficence of vigorous physical activity. Med Sci Sports Exerc 1985;17:94-100.

31. Rossier J, French ED, Rivier C, Ling N, Guillemin R, Bloom FE. Foot-shock induced stress increases betaendorphin levels in blood but not brain. Nature 1977;270:618-20.

32. Chung JS, Chong SY, Myoung JS, Choi HJ. Effect of physical exercise on plasma beta-endorphin level and pain threshold. J Korean Acad Rehabil Med 1993;17:368-73.

33. Al-Hasani R, Bruchas MR. Molecular mechanisms of opioid receptor-dependent signaling and behavior. Anesthesiology 2011;115:1363-81.

34. Koneru A, Satyanarayana S, Rizwan S. Endogenous opioids: their physiological role and receptors. Global J Pharmacol 2009;3:149-53.

35. McNicol E, Horowicz-Mehler N, Fisk RA, Bennett K, Gialeli-Goudas M, Chew PW, et al. Management of opioid side effects in cancer-related and chronic noncancer pain: a systematic review. J Pain 2003;4:231-56.

36. Millan MJ. Descending control of pain. Prog Neurobiol 2002;66:355-474.

37. Ren K, Dubner R. Descending modulation in persistent pain: an update. Pain 2002;100:1-6.

38. Fields HL, Basbaum AI, Heinricher MM. Central nervous system mechanisms of pain modulation. In: McMahon SB, Koltzenburg M, editors. Wall and Melzack's textbook of pain. 5th ed. Philadelphia: Elsevier/ Churchill Livingstone; 2006. p.125-42.

39. Jaggi AS, Singh N. Role of different brain areas in peripheral nerve injury-induced neuropathic pain. Brain Res 2011;1381:187-201.

40. Marinelli S, Vaughan CW, Schnell SA, Wessendorf MW, Christie MJ. Rostral ventromedial medulla neurons that project to the spinal cord express multiple opioid receptor phenotypes. J Neurosci 2002;22:1084755.

41. Smith MA, Yancey DL. Sensitivity to the effects of opioids in rats with free access to exercise wheels: muopioid tolerance and physical dependence. Psychopharmacology (Berl) 2003;168:426-34.

42. Bian D, Nichols ML, Ossipov MH, Lai J, Porreca F. Characterization of the antiallodynic efficacy of morphine in a model of neuropathic pain in rats. Neuroreport 1995;6:1981-4.

43. Takada T, Yamashita A, Date A, Yanase M, Suhara Y, Hamada A, et al. Changes in the circadian rhythm of mRNA expression for $\mu$-opioid receptors in the periaqueductal gray under a neuropathic pain-like state. Synapse 2013;67:216-23. 